\title{
Fish consumption and resilience to depression in Japanese company workers: a cross-sectional study
}

Eisho Yoshikawa ${ }^{1,2}$, Daisuke Nishi ${ }^{3^{*}}$ and Yutaka Matsuoka ${ }^{3}$

\begin{abstract}
Background: Depression is a common disorder that is influenced by psychosocial factors in the workplace. Increasing resilience, the ability to cope with stress in the face of adversity, is considered an important strategy to prevent depression. It has been suggested that consumption of fish, which is a major source of long chain n-3 polyunsaturated fatty acids (LC n-3 PUFA), may prevent depression. However, associations between depression, resilience, and fish consumption have not been documented.

The aim of the study is to investigate the association between fish consumption and resilience to depression.

Methods: Participants were 527 Japanese employees at three worksites of a large company. The Center for Epidemiologic Studies Depression (CES-D) Scale was administered to assess depressive symptoms, and the 14-item Resilience Scale (RS-14) was administered to assess resilience. A self-report questionnaire extracted from the Food Frequency Questionnaire was used to measure fish consumption frequency. Regression analyses were conducted to assess a mediation model based on a statistical analysis framework defined by Baron and Kenny. The indirect association of resilience was calculated with the bootstrapping method. Each analysis was adjusted by age, sex, marital status, work position, and educational background.

Results: The association between fish consumption frequency and total CES-D score was significant $(B=-0.94$; $p=0.011)$. The association between fish consumption frequency and total RS-14 score was significant $(B=1.4$; $p=0.010)$, as was association total RS-14 score and the total CES-D score $(B=-0.34 ; p<0.001)$. When controlling for total RS-14 score, there was no longer a significant association between fish consumption frequency and total CES-D score. The bootstrapping results revealed that significant indirect association though fish consumption frequency and total CES-D score (bias corrected and accelerated confidence interval $=-0.83$ to $-0.13 ; 95 \%$ confidence interval) through total RS-14 score.

Conclusions: Fish consumption might be associated with resilience to depression. Further studies are needed, particularly double blind randomized placebo controlled intervention trials on the potential preventative effect of LC n-3 PUFA on resilience to depression.
\end{abstract}

Keywords: Long chain n-3 poly unsaturated fatty acids, Fish consumption, Depression, Resilience

\section{Background}

Depression is a common disorder in the workplace and can result in impaired job performance [1], long absences due to sickness [2], and the need to pay disability pension [3]. The resulting loss due to reduced work capacity has been estimated to account for around one working month per ill worker per year [4]. Depression therefore represents a measurable economic burden to society [5, 6]. Adverse psychosocial factors, such as job strain (high demand and low decision latitude in the workplace) may be related to an elevated risk of subsequent depressive symptoms or major depressive episodes [7], and effective prevention strategies against depression are particularly important to both employees and employers, as well as society as a whole.

The attribute of resilience has garnered considerable attention in efforts to prevent mental disorders. Resili-

\footnotetext{
* Correspondence: d-nishi@umin.ac.jp

${ }^{3}$ Department of Psychiatry, National Disaster Medical Center, Tokyo, Japan

Full list of author information is available at the end of the article
} 
ence is defined as a dynamic process of adaptation to challenging life conditions $[8,9]$, and it has generally been viewed as the ability to cope with stress in the face of adversity [10]. Resilient persons tend to manifest adaptive behaviors [8], experience positive emotions even in stressful situations [11], and express emotional flexibility naturally in response to rapidly changing events [12]. Resilience has been associated with regulation of emotions and negatively associated with depression $[11,13]$. Increasing resilience is therefore viewed to be an important strategy to prevent depression [14].

Epidemiological evidence has shown a negative association between depression and fish consumption. For example, Hibbeln found a strong negative association between the two factors in 13 countries [15], and Tanskanen et al. found a higher prevalence of depression in low fish consumers than in high fish consumers in Finland [16]. Timosen et al. reported a higher risk of depression in women who ate fish rarely compared with those who ate fish more frequently [17]. Fish is rich source of long chain n-3 polyunsaturated fatty acids (LC n-3 PUFA), namely eicosapentaenoic acid (EPA) and docosahexaenoic acid (DHA). In human, EPA and DHA can be synthesized from Alphalinolenic acid (ALA), which is one of the two essential fatty acid that must be obtained from diets [18]. However the synthesis from ALA to DHA appears to be minimal [18] and there are evidences that DHA may be a semi-essential nutrient [19], which should be consumed directly from diet. There is high amount of LC n-3 PUFA in the brain and important structural component of the brain cell membrane. Parletta, et al. proposed six key mechanisms of the LC n-3 PUFA on the mental health as follow, 1) playing important role of neurite growth, 2) increasing fluidity and flexibility of cell membrane of the brain, 3) improving the function of neurotransmitter such as serotonergic and dopaminergic system 4) playing important role in endothelial function which may improve blood flow and blood brain barrier integrity via anti-inflammatory, vasodilatory, and increasing glucose transport 5) to encourage neuronal survival via increasing synthesis PS-DHA 6) to prevent neurodegeneration through increasing synthesis of neuroprotectin D1(NDP1) [20]. Therefore, LC n-3 PUFA may have important role for the regulation of mood and behavior. In addition, several meta-analyses have demonstrated the benefit of supplementation with LC n-3 PUFA for depression [21-24].

Given the above, frequent fish consumption may attenuate depressive symptoms by enhancing resilience. However, associations between fish consumption, resilience, and depression have not been well documented. The aim of this study was to investigate the association between fish consumption and resilience to depression.

\section{Results}

\section{Participant demographics}

All 527 participants were Japanese. The participants averaged $38.3 \pm 9.0$ years of age (range $=23-63$ ), 426 $(80.8 \%)$ were men, and 318 (60.3\%) were married. Regarding educational background, $456(85.5 \%)$ had graduated from college or university. Regarding working status, $41(7.8 \%)$ were in management positions, 58 (11.0 \%) held clerical jobs, 404 (76.7 \%) were engineers, and $15(2.7 \%)$ performed skilled work. Mean scores + Standard Deviation on the three parameters assessed were $63.5 \pm 11.0$ on the 14 -item Resilience Scale (RS-14), while Mean scores + Standard Deviation was $10.5 \pm 7.5$ on the Center for Epidemiologic Studies Depression (CES-D) Scale, and $3.0 \pm 0.9$ for the fish consumption score on the self-report questionnaire extracted from the Food Frequency Questionnaire (FFQ) on frequency of fish consumption. The score " $3.0 \pm 0.9$ " in the current study means that mean response of participants was 1-2 times per week of fish consumption, and mean - SD was 2.1 which meant about 1-3 times per month of fish consumption and mean + SD means was 3.9 which meant about 3-4 times per week of fish consumption.

\section{Univariate analysis}

The results of the univariate analyses are shown in Tables 1 and 2. Among the significant findings were that men ate fish more frequently than women, and married people had lower CES-D scores, higher RS-14 scores, and ate more fish than unmarried people (Tables 1 and 2). Those in management positions had higher RS-14 scores than those in non-management positions. The fish consumption frequency was positively associated with age, total CESD score and total RS-14 score.

\section{Mediation analysis}

The total mediator model was significant $F(7,519)=$ $30.45, p<0.0001$. The total explained variance $\left(R^{2}\right)$ was $29.1 \%$, and the adjusted $R^{2}$ was $28.2 \%$. As shown in Table 3, in the bootstrap analysis, the association between fish consumption frequency and total CES-D score was significant $(\mathrm{B}=-0.94 ; p=0.011)$. The association between fish consumption frequency and total RS-14 score was significant $(\mathrm{B}=1.4 ; p=0.010)$, as was association total RS-14 score and total CES-D score $(\mathrm{B}=-0.34 ; p<0.001)$. When controlling for total RS-14 score, there was no longer a significant relationship between fish consumption frequency and total CES-D score. The bootstrapping results revealed that significant indirect association though fish consumption frequency and total CES-D score (bias corrected and accelerated confidence inter$\mathrm{val}=-0.83$ to $-0.13 ; 95 \%$ confidence interval) through total RS-14 score. 
Table 1 Results of univariate analyses of the association of demographic categories on depression and resilience

\begin{tabular}{|c|c|c|c|c|c|c|c|c|c|c|}
\hline & & & \multicolumn{2}{|l|}{ CES-D } & & & \multicolumn{2}{|l|}{ RS-14 } & & \\
\hline & & & $r$ & $p$ & & & $r$ & $p$ & & \\
\hline \multirow[t]{2}{*}{ Age } & & & 0.039 & 0.368 & & & 0.078 & 0.07 & & \\
\hline & & $n$ & Mean & SD & $t$ & $p$ & Mean & SD & $t$ & $p$ \\
\hline \multirow[t]{2}{*}{ Sex } & male & 426 & 10.4 & 7.4 & -0.74 & 0.462 & 63.7 & 11.2 & 0.84 & 0.399 \\
\hline & female & 101 & 11.0 & 7.8 & & & 62.7 & 10.5 & & \\
\hline \multirow[t]{2}{*}{ Married } & yes & 318 & 9.4 & 6.8 & -4.27 & $<0.001$ & 65.1 & 10.4 & 4.26 & $<0.001$ \\
\hline & no & 209 & 12.3 & 8.2 & & & 61.0 & 11.5 & & \\
\hline \multirow[t]{2}{*}{ Graduated from university or college } & yes & 456 & 10.3 & 7.4 & -1.71 & 0.089 & 63.5 & 1.3 & -0.02 & 0.982 \\
\hline & no & 71 & 11.9 & 8.0 & & & 63.5 & 1.5 & & \\
\hline \multirow[t]{2}{*}{ Management position } & yes & 41 & 8.8 & 6.2 & -1.58 & 0.113 & 67.0 & 10.8 & 2.11 & 0.036 \\
\hline & no & 486 & 10.7 & 7.6 & & & 63.2 & 11.0 & & \\
\hline
\end{tabular}

CES-D Center for Epidemiologic Studies Depression, RS-14 14-item Resilience Scale, SD standard deviation

\section{Discussion}

This is the study to investigate the association between fish consumption and resilience to depression. Participants in current study replied that they usually ate fish or fish meals such as raw fish and/or grilled fish about 1-2 times per week on average. In univariate analysis, the older, married people, and men consumed fish more frequently, while males are more predominant (80\%) in this study. The result of the study suggested that fish consumption significantly associated with resilience to depression. Additionally, the results as analyzed with the statistical framework defined by Baron and Kenny [25], suggested that indirect association between fish consumption and depression thorough resilience. To best our knowledge there were few studies to investigate the association between fish consumption and resilience to depression, except for a study by Matsuoka and Nishi investigated the relationship between resilience and dietary factors, but not with depression, and found a positive association between higher fish consumption and resilience among rescue workers after the Great East Japan Earthquake [26].

Several lines of evidence suggest an association between resilience and emotional regulation. Tugade and Fredrickson examined cardiovascular reactivity in a stressful task and demonstrated that participants with higher resilience recovered more quickly from the cardiovascular arousal than participants with lower resilience, and this faster recovery was mediated by positive emotions [11]. Waugh demonstrated that participants

Table 2 Results of univariate analyses of the association between demographic characteristics, depressive symptoms, resilience and fish consumption frequency

\begin{tabular}{|c|c|c|c|c|c|}
\hline & & & \multicolumn{2}{|c|}{ Fish consumption frequency } & \\
\hline & & & rho & $p$ & \\
\hline Age & & & 0.153 & $<0.001$ & \\
\hline CES-D & & & -0.096 & 0.027 & \\
\hline RS-14 & & & 0.149 & $<0.001$ & \\
\hline & & $\mathrm{n}$ & mean & SD & $p$ \\
\hline Sex & male & 426 & 3.0 & 0.9 & 0.014 \\
\hline & female & 101 & 2.8 & 0.8 & \\
\hline Married & yes & 318 & 3.1 & 0.8 & $<0.001$ \\
\hline & no & 209 & 2.8 & 0.9 & \\
\hline Graduated from university or college & yes & 456 & 2.9 & 0.9 & 0.094 \\
\hline & no & 71 & 3.1 & 1.0 & \\
\hline Management position & yes & 41 & 2.9 & 0.9 & 0.952 \\
\hline & no & 486 & 3.0 & 0.9 & \\
\hline
\end{tabular}


Table 3 Results of mediation analysis and bootstrapping

\begin{tabular}{llllll}
\hline & B & SE & $t$ & $p$ & BCACl \\
\hline a path & $1.40^{\mathrm{a}}$ & 0.54 & 2.57 & 0.010 & - \\
b path & $-0.34^{\mathrm{a}}$ & 0.03 & -13.07 & $<0.001$ & - \\
c' path & $-0.47^{\mathrm{a}}$ & 0.32 & -1.47 & 0.142 & - \\
c path & $-0.94^{\mathrm{a}}$ & 0.37 & -2.57 & 0.011 & - \\
(c - c') path $^{\prime}$ & -0.47 & 0.18 & & & -0.83 to -0.13
\end{tabular}

SE Standard error, $B C A C l$ the bias corrected and assessed confidence intervals ${ }^{a}$ Regression coefficient between the pair of variables at each end of the indicated path (see Fig. 1)

with higher resilience show more appropriate and flexible emotional regulation in response to an anticipated threat than those with lower resilience [12, 27]. A functional magnetic resonance imaging study indicated that when anticipating a threat, the anterior insula, a region associated with emotional regulation, was activated for a shorter time in highly resilient participants than in those with low resilience [28].

Furthermore, several studies have associated the regulation of emotion in stressful situation with n-3 PUFA intake. Rats that experienced an early stressful situation of maternal separation showed less capacity to control fear responses when they lacked dietary n-3 PUFA [29]. Hamazaki et al. reported that DHA intake by university students prevented aggression against others from increasing at times of mental stress during the students' busiest and most frustrating days [30]. However, they did not find an effect on aggression in the absence of a stressful situation [31]. Suzuki et al. [32] found that intake of $\alpha$-linolenic acid and total n-3 PUFA might be associated with decreased incidence of depression in response to newly diagnosed lung cancer. Chang et al. [33] found that moderate depression, not severe, with cardiovascular disease had lower levels of DHA, n3PUFA, and $n 6 / n 3$ ratio in erythrocyte membranes compared with non-depression, while there were no difference in the electrocardiac markers and inflammation marker. Nishi et al. reported that fish oil supplementation attenuated posttraumatic symptoms in female rescue workers after the Great East Japan Earthquake [34]. Hence, fish consumption might promote the biological basis of appropriate and flexible emotional regulation in stressful situations, thus enhancing resilience.

Several studies have also suggested a possible preventive effect of LC n-3 PUFA supplementation on bipolar disorder [35], psychotic disorder [36], posttraumatic stress disorder following accidental injury [37], and interferon $\alpha$-induced depression [38]. Dietary modification is widely recognized and promoted for the primary prevention of non-communicable disorders, such as cardiovascular disease, obesity, and diabetes. Although strategies for preventing depression have traditionally focused on pharmacological and psychological approaches,
O'Neil et al. recently designed a study to investigate the effect of an individualized, structured dietary intervention on depression [39]. In addition, several approaches that increase resilience, such as well-being therapy, are used to treat depression $[14,40]$, by not only attenuating and preventing negative symptoms but also promoting positive emotions in order to increase psychological well-being [14]. Such a psychotherapeutic approach may increase resilience and prevent recurrence of depression [41, 42]. The findings of current study cannot deny the possibility that fish consumption increase the resilience and prevent depression, but this warrants further investigation.

\section{Conclusion and limitations}

Fish consumption might be associated with resilience to depression in Japanese company workers. However, further studies are needed, particularly double blind randomized placebo controlled intervention trials on the potential preventative effect of LC n-3 PUFA on resilience to depression.

Our study had several limitations. First, the participants were mainly men, they were highly educated, and they worked for a big Japanese company that provides good job security and a relatively good balance of effort and reward. Therefore, they might not be representative of workers more generally. Second, this study was conducted in Japan, a country already known to have very high levels of fish consumption, which is likely to have influenced LC n-3 PUFA levels in comparison with other populations. Third, information on fish intake frequency was self-reported, and non-differential misclassification may be inevitable and could attenuate the observed associations. Furthermore, specific food frequency questionnaire for LC n-3 PUFA intakes was not used in this study. It was reported that a specific food frequency questionnaire to assess LC n-3 PUFA intakes was superior to a generic FFQ [43]. Therefore, we may not be able to assess LC n-3 PUFA intakes in this study appropriately. In addition, no other dietary ingredients that might affect resilience were evaluated in this study. Fourth, due to the cross-sectional nature of the study design, causal relationships between the factors could not be determined. Finally, residual confounding by uncontrolled or unmeasured factors may have distorted genuine associations.

\section{Methods}

\section{Participants and procedures}

This study was approved by the institutional review boards of both the National Disaster Medical Center and the company. This study was conducted using data collected in a previous study [44]. A cross-sectional study was carried out at three separate worksites of a large company located in an urban area of Japan between August and November 2010. The inclusion criteria were as 
follows: company worker, age $\geq 18$ years, and capable of understanding and providing consent for study participation. The company's occupational health staff provided participants with a written explanation of the research, a consent form, and the self-reporting questionnaire. Workers who agreed to participate in this study provided consent by returning the consent form and questionnaire by postal mail.

Of the 6204 workers at the three sites of the company, 807 workers (13\%) were approached. Among them, 538 $(66.7 \%)$ agreed to participate in the study. We excluded 11 participants with missing responses to items related to the subscales used, leaving 527 participants for analysis in this study. The workers who did not participate did not differ significantly from the participants in terms of age or sex.

\section{Measures}

\section{Demographics}

We gathered information on sex, marital status (married or not), educational background (graduated from college or university or not), and job status (management position or not).

\section{Assessment of fish consumption in daily life}

To evaluate fish consumption in daily life, we extracted one items from the short version of the FFQ [45]. Fish consumption was assessed with a frequency question: "How often do you usually eat fish or fish meals such as Sashimi (raw fish) and/or Yakizakana (grilled fish)? Please consider the last six months." Six response options were given for each question: almost none, 1-3 times/month, 1-2 times/ week, 3-4 times/week, 5-6 times/week, and every day.

\section{Assessment of depressive symptoms}

We administered the CES-D to assess depressive symptoms. This scale is one of the most widely used scales to assess depressive symptoms in the general population and measures the level of depressive symptoms in the past week [46]. The CES-D is composed of 20 items, and the scores are summed to yield a total score between 0 and 60 , with a higher score indicating more severe depression. The reliability and validity of the Japanese version have been verified [47].

\section{Assessment of resilience}

The Resilience Scale (RS) is a self-reported questionnaire consisting of 25 items that measure the degree of

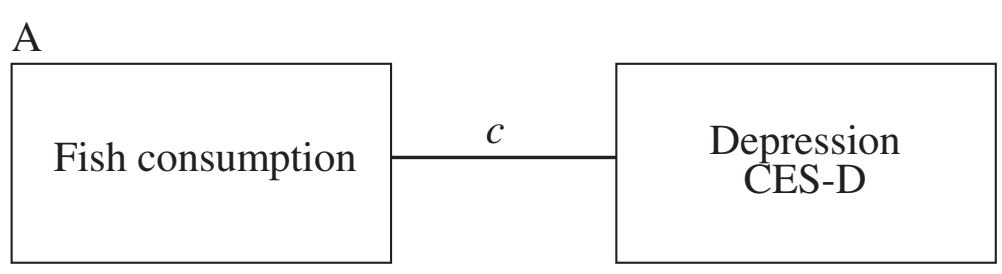

B

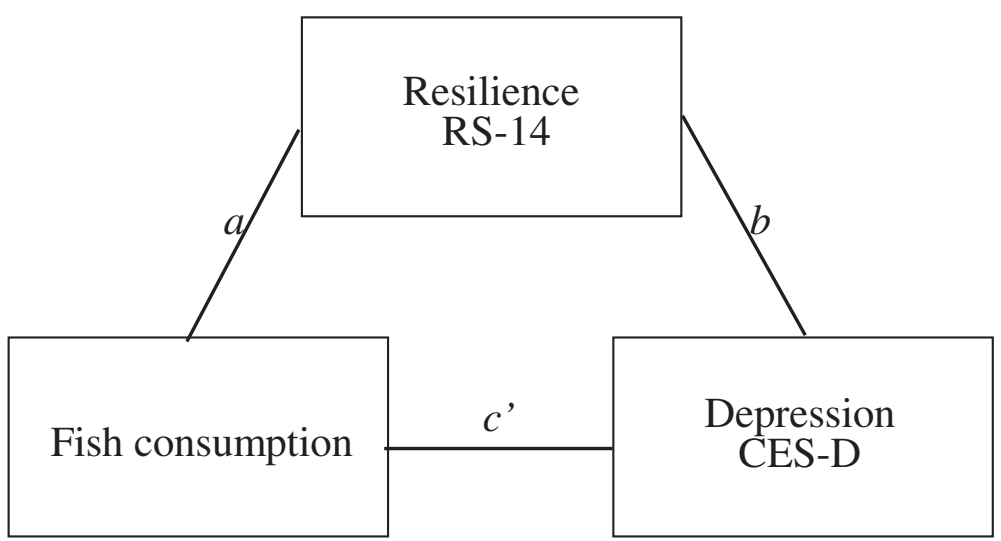

Fig. 1 a lllustration of a direct association of the dietary factor of fish consumption on depression. Path c represents the total association of fish consumption on depression (CES-D). $\mathbf{b}$ Illustration of an indirect association between fish consumption and depression (CES-D) through resilience (RS-14). Path a represents the association between fish consumption and resilience (RS-14), the proposed mediator. Path $b$ represents the association between resilience (RS-14) and depression (CES-D), without fish consumption. Path $\mathrm{C}^{\prime}$ is the association between the fish consumption and depression (CES-D), without resilience (RS-14). The indirect association between the fish consumption and depression (CES-D) through resilience (RS-14) score is C $-c^{\prime}$, which is tested with the confidence interval obtained through the bootstrapping method 
individual resilience [8]. The RS was developed based on a qualitative study of 24 older women in America who had experienced a recent loss (e.g., of a spouse, health, or employment) and had adapted successfully [8, 48-52]. Its conceptual framework is composed of five characteristics. Items are categorized by (i) self-reliance (e.g. 'I feel I can handle many things at a time'); (ii) meaning (e.g. 'I feel proud that I have accomplished things in life'); (iii) equanimity (e.g. 'I usually take things in stride'); (iv) perseverance (e.g. 'I am determined'); and (v) existential aloneness (e.g. 'my belief in myself gets me through hard times'). The shorter RS-14 version, consisting of 14 items, strongly correlates with the RS. Each item is rated on a 7-point Likert scale (range, 14-98), with a higher score indicating more resilience [8]. The reliability and validity of the Japanese version have been verified (10).

\section{Statistical analysis Univariate analysis}

Two-tailed Pearson's correlations were used to examine the intercorrelation between age, total CES-D score, and total RS-14 score. Spearman's rank correlation test was used to examine the intercorrelation between fish consumption frequency and age, total CESD score, and total RS-14 score. Student's $t$-test was used to compare CES-D and RS-14 scores between demographic categories. The Mann-Whitney $U$ test was used to compare fish consumption frequency between demographic categories.

\section{Mediation analysis}

We investigate the indirect association between the fish consumption frequency and total CESD score through the total RS-14 scores illustrated (Fig. 1a and b). Indirect association is assessed by comparing the total association $(c)$ of an independent variable on a dependent variable, which is composed of a direct association $\left(c^{\prime}\right)$ of the independent variable on the dependent variable and an indirect association $\left(c-c^{\prime}\right)$ of the independent variable on the dependent variable through a proposed mediation variable. Coefficient $a$ represents the association of the independent variable on the mediation variable $M$, whereas coefficient $b$ represents the association of $M$ on the dependent variable. According to the statistical analysis framework defined in [25], mediation models require that $a, b$, and $c$ are significant and that $c^{\prime}$ is smaller than $c$ by a nontrivial amount [53].

Three regression analysis were conducted to assess the mediation model in the statistical analysis framework defined by Baron and Kenny [25]. Each analysis was adjusted by age, sex, marital status, employment position, and educational background. The first regression analysis was conducted to evaluate the $c^{\prime}$ path, with total CES-D score as the dependent variable and fish consumption frequency as the independent variable. The second regression analysis was conducted to evaluate the $a$ path, with total RS-14 score as the dependent variable and fish consumption frequency as the independent variable. The third regression analysis, which represented the total mediator model, was conducted to evaluate the $b$ and $c$ paths, with total CES-D score as the dependent variable and fish consumption frequency and total RS-14 score as independent variables.

Next, the indirect association was calculated from the unstandardized regression weights of paths $a$ and $b$ [25]. Bootstrapping was used to produce the sampling distributions of the indirect association by sampling from the data set (in this case, $n=5000$ samples) and calculating the indirect association present in the resamples.

All of the analyses were performed using SPSS, version 21 (SPSS Inc., Chicago) and Preacher and Hayes' bootstrap script for SPSS [54]. BCACI was set at $95 \%$. The use of a $95 \%$ confidence interval is equivalent to testing for significance at the .05 level.

\section{Abbreviations \\ LC n-3 PUFA: Long chain n-3 polyunsaturated fatty acids; FFQ: Food Frequency Questionnaire; CES-D: The Center for Epidemiologic Studies Depression; RS-14: 14-item Resilience Scale; Cl: Confidence interval; $\mathrm{BCACl}$ : Bias corrected and accelerated confidence interval.}

\section{Competing interests}

The authors alone are responsible for the content and writing of the paper.

\section{Authors' contributions}

All authors participated in the study to a significant extent and worked on study conception and design; data collection, analysis, and interpretation; and writing the manuscript. All authors contributed to the intellectual content of the article and read and approved the submitted manuscript.

\section{Acknowledgments}

The authors thank Koichi Iwata and Toshinari Saeki for their cooperation with the research. We also express special thanks to Mss. Akutsu and Kamoshida for data management and to all participants in this study. This work was supported by grants from the Foundation for Total Health Promotion, and CREST Japan Science and Technology Agency.

Dr. Yoshikawa has received research support from a Research Promotion Grant of Nippon Medical School and lecture fees from Dainippon

Pharmaceutical Co., Ltd. and Mochida Pharmaceutical Co., Ltd. Dr. Nishi has received research support from the Japan Society for the Promotion of Science and lecture fees from Qol Co., Ltd., DHA \& EPA Association, NTT DoCoMo, Inc., Emotional Quotient Academy, Ltd., and Otsuka Pharmaceutical Co., Ltd. Dr. Matsuoka has received research support from the Japan Science and Technology Agency, CREST, and the Ministry of Health, Labor and Welfare of Japan, an Intramural Research Grant for Neurological and Psychiatric Disorders from the National Center of Neurology and Psychiatry, and lecture fees from Ono Pharmaceutical Co. Ltd., Mochida Pharmaceutical Co. Ltd., Takeda Pharmaceutical Company Ltd., Suntory Wellness Ltd., DHA \& EPA Association, and Otsuka Pharmaceutical Co., Ltd.

\section{Author details}

'Department of Neuropsychiatry, Nippon Medical School Tama Nagayama Hospital, Tokyo, Japan. ${ }^{2}$ Department of Neuropsychiatry, Nippon Medical School, Tokyo, Japan. ${ }^{3}$ Department of Psychiatry, National Disaster Medical Center, Tokyo, Japan.

Received: 10 November 2014 Accepted: 15 May 2015

Published online: 26 May 2015 


\section{References}

1. Adler DA, McLaughlin TJ, Rogers WH, Chang H, Lapitsky L, Lerner D. Job performance deficits due to depression. Am J Psychiatry. 2006;163:1569-76.

2. Bültmann U, Rugulies $R$, Lund $T$, Christensen $K B$, Labriola M, Burr $H$. Depressive symptoms and the risk of long-term sickness absence: a prospective study among 4747 employees in Denmark. Soc Psychiatry Psychiatr Epidemiol. 2006;41:875-80.

3. Mykletun A, Overland S, Dahl AA, Krokstad S, Bjerkeset O, Glozier N, et al. A population-based cohort study of the effect of common mental disorders on disability pension awards. Am J Psychiatry. 2006;163:1412-8.

4. Kessler RC, Akiskal HS, Ames M, Birnbaum H, Greenberg P, Hirschfeld RMA, et al. Prevalence and effects of mood disorders on work performance in a nationally representative sample of U.S. workers. Am J Psychiatry. 2006:163:1561-8.

5. Luppa M, Heinrich S, Angermeyer MC, König H-H, Riedel-Heller SG. Cost-ofillness studies of depression: a systematic review. J Affect Disord. 2007:98:29-43.

6. Cuijpers P, Smit F, Oostenbrink J, de Graaf R, Ten Have M, Beekman A. Economic costs of minor depression: a population-based study. Acta Psychiatr Scand. 2007;115:229-36.

7. Bonde JPE. Psychosocial factors at work and risk of depression: a systematic review of the epidemiological evidence. Occup Environ Med. 2008:65:438-45.

8. Wagnild GM, Young HM. Development and psychometric evaluation of the Resilience Scale. J Nurs Meas. 1993;1:165-78.

9. Gartland D, Bond L, Olsson CA, Buzwell S, Sawyer SM. Development of a multi-dimensional measure of resilience in adolescents: the Adolescent Resilience Questionnaire. BMC Med Res Methodol. 2011;11:134.

10. Nishi D, Uehara R, Kondo M, Matsuoka Y. Reliability and validity of the Japanese version of the Resilience Scale and its short version. BMC Res Notes. 2010;3:310.

11. Tugade Michele M, Fredrickson BL. Resilient individuals Use positive emotions to bounce back from negative emotional experiences. J Pers Soc Psychol. 2004;86:320-33.

12. Waugh CE, Thompson RJ, Gotlib IH. Flexible emotional responsiveness in trait resilience. Emotion. 2011:11:1059-67.

13. Beasley M, Thompson T, Davidson J. Resilience in response to life stress: the effects of coping style and cognitive hardiness. Pers Individ Dif. 2003;34:77-95.

14. Waugh CE, Koster EHW. A resilience framework for promoting stable remission from depression. Clin Psychol Rev. 2014, doi.org/10.1016/ j.cpr.2014.05.004

15. Hibbeln JR. Fish consumption and major depression. Lancet. 1998;351:1213.

16. Tanskanen A, Hibbeln JR, Tuomilehto J, Uutela A, Haukkala A, Viinamäki H, et al. Fish consumption and depressive symptoms in the general population in Finland. Psychiatr Serv. 2001;52:529-31.

17. Timonen M, Horrobin D, Jokelainen J, Laitinen J, Herva A, Räsänen P. Fish consumption and depression: The Northern Finland 1966 birth cohort study. J Affect Disord. 2004:82:447-52.

18. Burdge G. Alpha-linolenic acid metabolism in men and women: nutritional and biological implications. Curr Opin Clin Nutr Metab Care. 2004;7:137-44.

19. Vlaardingerbroek $H$, Hornstra G. Essential fatty acids in erythrocyte phospholipids during pregnancy and at delivery in mothers and their neonates: Comparison with plasma phospholipids. Prostaglandins Leukot Essent Fat Acids. 2004;71:363-74

20. Parletta N, Milte CM, Meyer BJ. Nutritional modulation of cognitive function and mental health. J Nutr Biochem. 2013;24:725-43.

21. Appleton KM, Rogers PJ, Ness AR. Updated systematic review and metaanalysis of the effects of $n-3$ long-chain polyunsaturated fatty acids on depressed mood. Am J Clin Nutr. 2010;91:757-70.

22. Bloch MH, Hannestad J. Omega-3 fatty acids for the treatment of depression: systematic review and meta-analysis. Mol Psychiatry. 2012;17:1272-82.

23. Sublette ME, Ellis SP, Geant AL, Mann JJ. Meta-analysis: effects of eicosapentaenoic acid in clinical trials in depression. J Clin Psychiatry. 2013;72:1577-84.

24. Grosso G, Pajak A, Marventano S, Castellano S, Galvano F, Bucolo C, et al. Role of omega-3 fatty acids in the treatment of depressive disorders: a comprehensive meta-analysis of randomized clinical trials. PLoS One. 2014;9, e96905.

25. Baron RM, Kenny DA. The moderator-mediator variable distinction in social psychological research: conceptual, strategic, and statistical considerations. J Pers Soc Psychol. 1986;51:1173-82.
26. Matsuoka Y, Nishi D. The possiblity of omega-3 fatty acid improving resilience. Japnese J Gen Hosp psychiatry. 2012;24:25-32.

27. Waugh CE, Fredrickson BL, Taylor SF. Adapting to life's slings and arrows: Individual differences in resilience when recovering from an anticipated threat. J Res Pers. 2008;42:1031-46.

28. Waugh CE, Wager TD, Fredrickson BL, Noll DC, Taylor SF. The neura correlates of trait resilience when anticipating and recovering from threat. Soc Cogn Affect Neurosci. 2008;3:322-32.

29. Mathieu G, Oualian C, Denis I, Lavialle M, Gisquet-Verrier P, Vancassel S. Dietary n-3 polyunsaturated fatty acid deprivation together with early maternal separation increases anxiety and vulnerability to stress in adult rats. Prostaglandins Leukot Essent Fatty Acids. 2011;85:129-36.

30. Hamazaki T, Sawazaki S, Itomura M, Asaoka E, Nagao Y, Nishimura N, et al. The effect of docosahexaenoic acid on aggression in young adults: A placebo-controlled double-blind study. J Clin Invest. 1996;97:1129-33.

31. Hamazaki T, Sawazaki S, Nagao Y, Kuwamori T, Yazawa K, Mizushima Y, et al. Docosahexaenoic acid does not affect aggression of normal volunteers under nonstressful conditions. A randomized, placebo-controlled, DoubleBlind Study. Lipids. 1998;33:663-7.

32. Suzuki S, Akechi T, Kobayashi M, Taniguchi K, Goto K, Sasaki S, et al. Daily omega-3 fatty acid intake and depression in Japanese patients with newly diagnosed lung cancer. Br J Cancer. 2004;90:787-93.

33. Chang JP-C, Chang S-S, Yang H-T, Palani M, Chen C-P, Su K-P. Polyunsaturated fatty acids (PUFAs) levels in patients with cardiovascular diseases (CVDs) with and without depression. Brain Behav Immun. 2015;44:28-31.

34. Nishi D, Koido $Y$, Nakaya $N$, Sone $T$, Noguchi $H$, Hamazaki $K$, et al. Fish oil for attenuating posttraumatic stress symptoms among rescue workers after the great east Japan earthquake: a randomized controlled trial. Psychother Psychosom. 2012:81:315-7.

35. Stoll AL, Severus WE, Freeman MP, Rueter S, Zboyan HA, Diamond E, et al. Omega 3 fatty acids in bipolar disorder a preminary double-blind, placebocontrolled trial. Arch Gen Psychiatry. 1999;56:407-12.

36. Amminger GP, Schäfer MR, Papageorgiou K, Klier CM, Cotton SM, Harrigan SM, et al. Long-chain omega-3 fatty acids for indicated prevention of psychotic disorders: a randomized, placebo-controlled trial. Arch Gen Psychiatry. 2010;67:146-54.

37. Matsuoka Y, Nishi D, Yonemoto N, Hamazaki K, Hashimoto K, Hamazaki T. Omega-3 fatty acids for secondary prevention of posttraumatic stress disorder after accidental injury: an open-label pilot study. J Clin Psychopharmacol. 2010:30:217-9.

38. Su K-P, Lai H-C, Yang H-T, Su W-P, Peng C-Y, Chang JP-C, et al. Omega-3 fatty acids in the prevention of interferon-alpha-induced depression: results from a randomized, controlled trial. Biol Psychiatry. 2014;76:559-66.

39. O'Neil A, Berk M, Itsiopoulos C, Castle D, Opie R, Pizzinga J, et al. A randomised, controlled trial of a dietary intervention for adults with major depression (the "SMILES" trial): study protocol. BMC Psychiatry. 2013;13:114

40. Fava GA, Tomba E. Increasing psychological well-being and resilience by psychotherapeutic methods. J Pers. 2009;77:1903-34.

41. Fava GA, Ruini C, Rafanelli C, Finos L, Conti S, Grandi S. Six-year outcome of cognitive behavior therapy for prevention of recurrent depression. Am J Psychiatry. 2004:161:1872-6.

42. Teasdale JD, Segal ZV, Williams JM, Ridgeway VA, Soulsby JM, Lau MA. Prevention of relapse/recurrence in major depression by mindfulness-based cognitive therapy. J Consult Clin Psychol. 2000;68:615-23.

43. Meyer BJ, Swierk M, Russell KG. Assessing long-chain $\omega-3$ polyunsaturated fatty acids: a tailored food-frequency questionnaire is better. Nutrition. 2013;29:491-6.

44. Nishi D, Uehara R, Yoshikawa E, Sato G, Ito M, Matsuoka Y. Culturally sensitive and universal measure of resilience for Japanese populations: Tachikawa Resilience Scale in comparison with Resilience Scale 14-item version. Psychiatry Clin Neurosci. 2013:67:174-81.

45. Tsubono Y, Takamori S, Kobayashi M, Takahashi T, Iwase YIY. A data-based questionnaire approach for designing a semiquantitative prospective study food in Japan frequency for a population-based prospective study in Japan. J Epidemiol. 1996;6:45-53.

46. Radloff LS. The CES-D Scale: a self-report depression scale for research in the general population. Appl Psychol Meas. 1977;1:385-401.

47. Shima S, Shikano T, Kitamura TAM. A new selfreport depression scale. Seishinigaku. 1985:27:717-23.

48. Wagnild GM. The Resilience Scale User's Guide for the US English Version of the Resilience Scale and the 14-Item Reselience Scale (RS-14). Montana: The Resilience Center; 2009. 
49. Abiola T, Udofia O. Psychometric assessment of the Wagnild and Young's resilience scale in Kano, Nigeria. BMC Res Notes. 2011;4:509.

50. Perna L, Mielck A, Lacruz ME, Emeny RT, Holle R, Breitfelder A, et al. Socioeconomic position, resilience, and health behaviour among elderly people. Int J Public Health. 2012;57:341-9.

51. Salazar-Pousada D, Arroyo D, Hidalgo L, Pérez-López FR, Chedraui P. Depressive symptoms and resilience among pregnant adolescents: a case-control study. Obstet Gynecol Int. 2010;2010:952493.

52. Damásio BF, Borsa JC, da Silva JP. 14-item resilience scale (RS-14): psychometric properties of the Brazilian version. J Nurs Meas. 2011;19:131-45.

53. James LR, Brett JM. Mediators, moderators, and tests for mediation. J Appl Psychol. 1984;69:307-21.

54. Preacher KJ, Hayes AF. Asymptotic and resampling strategies for assessing and comparing indirect effects in multiple mediator models. Behav Res Methods. 2008:40:879-91.

\section{Submit your next manuscript to BioMed Central and take full advantage of:}

- Convenient online submission

- Thorough peer review

- No space constraints or color figure charges

- Immediate publication on acceptance

- Inclusion in PubMed, CAS, Scopus and Google Scholar

- Research which is freely available for redistribution 